\title{
Câncer pediátrico: análise de um registro hospitalar
}

\author{
Pediatric cancer: analysis of hospital records
}

\author{
Denise Bousfield da Silva ${ }^{1}$, Maria Marlene de Souza Pires ${ }^{2}$, Silvia Modesto Nassar ${ }^{3}$
}

\section{Resumo}

Objetivo: analisar um registro hospitalar de câncer pediátrico, descrevendo a ocorrência de casos novos; verificar a relação com sexo, idade, raça e extensão clínica da doença; relatar a mortalidade das crianças com câncer; explorar a associação de casos novos com as variáveis demográficas propostas, extensão clínica da doença e status vital.

Método: estudo observacional, descritivo, longitudinal em crianças até a idade de 14 anos completos, procedentes de Santa Catarina. As 371 crianças portadoras de casos novos de câncer foram atendidas no ambulatório de referência regional entre 1994 e 1998, e registradas segundo a Classificação Internacional do Câncer na Infância. Foi aplicada a análise de correspondência múltipla para avaliar as variáveis categóricas.

Resultados: foram registrados 371 casos novos de câncer, com distribuição constante por ano, no período estudado. O câncer ocorreu no pré-escolar em $41,5 \%$, e no sexo masculino em $55,8 \%$. A leucemia foi observada em $36,6 \%$. O estádio não localizado da doença na ocasião do diagnóstico ocorreu em 55,7\%. Foram a óbito $55,7 \%$ dos casos com doença não localizada, e $16,3 \%$ com doença localizada. No término do estudo, $58,8 \%$ das crianças estavam vivas.

Conclusões: observa-se predomínio do câncer no pré-escolar e no sexo masculino. A leucemia é o câncer mais freqüente. A doença não localizada predomina na ocasião do diagnóstico. A freqüência de óbito é mais elevada no grupo com doença não localizada. Há associação direta com doença localizada, status vital vivo, escolar, adolescente e sexo feminino; e para doença não localizada, com óbito, lactente, pré-escolar e sexo masculino.

$J$ Pediatr (Rio J) 2002; 78 (5): 409-14: neoplasias, pediatria, registros hospitalares, epidemiologia descritiva, análises descritivas e exploratórias.

\section{Introdução}

O câncer na criança representa aproximadamente 2\% das neoplasias malignas. Constitui-se em uma das princi-

1. Professora do Dep. de Pediatria da Univ. Federal de Santa Catarina (UFSC), Mestre em Ciências Médicas pela UFSC, Médica do Serviço de Oncohematologia do Hospital Infantil Joana de Gusmão (HIJG), Florianópolis, SC.

2. Professora Dra. do Departamento de Pediatria da UFSC, Coordenadora do Serviço de Metabologia e Nutrição do HIJG, Florianópolis, SC.

3. Professora Dra. do Departamento de Informática da UFSC.

Artigo submetido em 06.03.02, aceito em 19.06.02.

\begin{abstract}
Objective: to analyze pediatric cancer hospital records regarding the occurrence of new cases; to verify the relation between sex, age, race, origin and the disease clinical extension; to describe the mortality of children with cancer; to explore the association of new cases with proposed demographic variables, disease clinical extension and vital status.
\end{abstract}

Methods: observational, descriptive, longitudinal study in children up to 14 years old, from Santa Catarina. Three hundred and seventy-one new cases of cancer were treated in a regional reference outpatient clinic (1994-98) and recorded according to the International Classification of Childhood Cancer. Multiple Correspondence Analysis was applied to evaluate the categorical variables.

Results: three-hundred and seventy-one new cases of cancer were recorded with constant distribution in each year. Cancer affected preschoolers $(41.5 \%)$ and males $(55.8 \%)$. Leukemia was observed in $36.6 \%$ of the cases. Non-localized stage on the diagnosis occurred in $55.7 \%$. The death occurred in $55.7 \%$ of the cases with non-localized disease and $16.3 \%$ with localized disease. At the end of the study $58.8 \%$ of the children were alive.

Conclusions: we observed more cases of cancer at pre-school age and among male subjects. Leukemia is the most frequent type of cancer. Non-localized disease predominates on the diagnosis. The death frequency is higher in the group with non-localized disease. There is direct association with non-localized disease, living vital status, school age, adolescent age, female, and for non-localized disease with death vital status, infant age, pre-school age and male.

J Pediatr (Rio J) 2002; 78 (5): 409-14: neoplasia, pediatrics, hospital records, descriptive epidemiology, descriptive and exploratory analysis.

pais causas de óbito por doença nesta faixa etária, e é responsável pela maior perda de potenciais anos de vida ${ }^{1}$.

Nas crianças, as neoplasias malignas diferem daquelas observadas nos adultos, tanto em relação a sua freqüência quanto ao seu tipo histológico. $O$ câncer na criança afeta as células do sistema hematopoiético e os tecidos de sustentação, enquanto que, no adulto, afeta as células do epitélio que recobrem os diferentes órgãos ${ }^{1-4}$.

O tratamento deve ser realizado por uma equipe interdisciplinar, em centros oncológicos especializados, e pla- 
nejado de acordo com o tipo histológico e com o estadiamento clínico do câncer ${ }^{5}$.

É prioritária a criação e padronização de centros de registro de câncer para a implementação das pesquisas clínicas e epidemiológicas. Os estudos epidemiológico e etiológico dos cânceres pediátricos são escassos, quando comparados aos do adulto ${ }^{3,6}$. Estes centros fornecem subsídios básicos para o estabelecimento de estratégias eficientes no atendimento ao paciente oncológico, e no aprimoramento de atividades relacionadas ao controle do câncer em termos de saúde pública ${ }^{6-9}$.

Os centros de registro devem adotar uma mesma classificação para que seja possível a realização de estudos comparativos nacionais e internacionais. Os objetivos são determinados pela sua natureza, ou seja, registros de câncer de base populacional (RCBP) e registros hospitalares de câncer (RHC) ${ }^{8,9}$.

O RCBP tem uma atividade mais complexa, e seu principal propósito é avaliar o impacto do câncer em uma determinada população $0^{3,10,11}$.

Os RHC coletam dados de todos os pacientes atendidos no hospital com diagnóstico confirmado de câncer, e visam, principalmente, o aprimoramento da assistência médica $^{8,10,12}$.

Este estudo se propõe a analisar um registro hospitalar de câncer pediátrico em um centro de referência de Santa Catarina, no que concerne à ocorrência de casos novos de neoplasias malignas primárias, em relação à idade, sexo, raça ou cor, extensão clínica da doença e status vital.

\section{Método}

Esta pesquisa foi um estudo observacional, descritivo e longitudinal, realizado no Hospital Infantil Joana de Gusmão (HIJG), da Secretaria de Estado de Saúde de Santa Catarina (SES-SC). Este é um Hospital-Escola, terciário, de referência regional no atendimento às neoplasias malignas na criança.

O estudo foi delineado de acordo com as diretrizes e normas regulamentadoras de pesquisas envolvendo seres humanos $^{13}$, e foi aprovado pela comissão de ética em pesquisa em seres humanos da Universidade Federal de Santa Catarina.

A população de estudo incluiu 371 crianças com idade de até 14 anos completos, procedentes de Santa Catarina, e diagnosticadas no período de janeiro de 1994 a dezembro de 1998. Estas crianças foram atendidas no ambulatório e enfermaria do serviço de oncohematologia (SHO) do HIJG, sendo acompanhadas clinicamente até julho de 2000.

A amostra incluiu todos os casos novos de neoplasias malignas primárias de qualquer topografia, e as de comportamento incerto do sistema nervoso central (SNC), de forma similar a outros estudos ${ }^{14-16}$

A confirmação diagnóstica foi efetuada utilizando-se exames citomorfológicos ou histopatológicos, além de estudos citoquímicos e/ou imunohistoquímicos ${ }^{17,18}$. As cri- anças com suspeita clínica bem fundamentada de malignidade, e com diagnóstico por imagem em área não acessível ao diagnóstico microscópico, também foram incluídas neste estudo. Considerou-se como critérios de exclusão a criança portadora de neoplasia maligna secundária ao tratamento quimioterápico ou radioterápico; criança com diagnóstico de neoplasia maligna, que chegou a este serviço para realizar uma modalidade terapêutica específica, e que retornou, posteriormente, ao serviço de origem; recidiva de tratamentos realizados em outros serviços e perda de seguimento.

A Classificação Internacional de Doenças para Oncologia (CID-O) foi o procedimento empregado para codificação da morfologia (histologia) ${ }^{19}$. A Classificação Internacional do Câncer na Infância (CICI), que o categoriza de acordo com a sua morfologia em doze principais grupos de diagnóstico, foi utilizada para tabulação e análise dos dados $^{20}$.

A extensão da doença foi avaliada através do estadiamento clínico para todos os grupos de diagnóstico, exceto para leucemia (não empregável) ${ }^{21}$.

A coleta dos dados foi realizada no período de junho a julho de 2000, nos prontuários do serviço de oncohematologia (SOH) do HIJG e do serviço de arquivo médico (SAME) do referido hospital, através de ficha apropriada.

As informações incompletas foram resgatadas pela pesquisadora e pelo serviço social por meio de contato pessoal ou telefônico com os pais, parentes, Secretarias de Saúde dos municípios envolvidos e com os cartórios de registro civil.

As variáveis utilizadas neste estudo foram: idade; sexo; cor ou raça (segundo classificação estabelecida pelo Instituto Brasileiro de Geografia e Estatística ${ }^{22}$, tipo histológico da neoplasia maligna primária (de acordo com a CICI) ${ }^{20}$, extensão clínica da neoplasia maligna primária (segundo o estadiamento clínico específico para cada tipo histológico) e status vital.

Foram consideradas neoplasia maligna ou câncer as neoplasias malignas primárias de qualquer topografia e as de comportamento incerto do SNC.

O termo comportamento incerto foi utilizado para definir as neoplasias com comportamento agressivo pela sua localização, porém em áreas não acessíveis ao diagnóstico histopatológico.

A ocorrência de casos novos foi o termo determinado para referir a freqüência de casos novos e, desta maneira, diferenciar do estudo de base populacional, o qual utiliza incidência.

O termo status vital foi empregado para definir a condição clínica da criança portadora de neoplasia maligna primária no término do estudo, e foi categorizado em: vivo, fora de tratamento; vivo, em tratamento; e óbito.

Os grupos etários foram categorizados segundo a classificação adotada pela Faculdade de Medicina da Universidade de São Paulo²3. 
A extensão clínica da neoplasia maligna primária foi definida de acordo com o estadiamento clínico, e determinada pela extensão da doença no local primário, presença de metástases regionais e à distância. Neste estudo, foi considerada doença localizada os estádios I e II, e doença não localizada, os estádios III e IV.

Os procedimentos estatísticos utilizados foram as medidas descritivas ${ }^{24}$, as tabelas de freqüência e análise de correspondência múltipla (ACM) ${ }^{25}$. A ACM foi empregada para investigar a existência de associação entre as variáveis categóricas: grupo etário, sexo, extensão clínica da doença e status vital. Quando se pretende estudar um fenômeno complexo, com múltiplas variáveis, cujas relações e inter-relações se quer conhecer, estão indicadas como ferramentas analíticas as análises multivariadas. A ACM é uma das técnicas de análise multivariada que possibilita avaliar simultaneamente um conjunto de variáveis categóricas, segundo sua intensidade e grau de associação entre elas. Os resultados estatísticos oriundos da ACM são: uma ampla tabela de freqüências, conhecida como tabela de Burt, e uma representação gráfica simplificada dos dados. Esta representação gráfica pode ser mostrada em dois ou três eixos ou dimensões. Em duas dimensões, podese observar o padrão de associação em plano relacional, e em três, em um espaço relacional. Da análise destes resultados, é possível identificar padrões de associações existentes entre as variáveis analisadas. A intensidade do padrão de associação está relacionada ao percentual de inércia alcançado neste procedimento, e que depende das características dos dados em análise. O percentual de inércia expressa o grau da variabilidade total dos dados, explicado pelo pa- drão de associação identificado e representado graficamente. À medida que se inclui uma nova dimensão na ACM, aumenta-se numericamente o percentual de inércia. A primeira dimensão é a de maior inércia ${ }^{25}$.

$\mathrm{Na} \mathrm{ACM}$, o status vital foi categorizado em vivo e óbito, devido à baixa freqüência de crianças vivas em tratamento.

\section{Resultados}

Foram registrados, no período de janeiro de 1994 a dezembro de 1998, 371 casos novos de crianças portadoras de neoplasias malignas primárias. A perda de seguimento ocorreu em cinco casos. A casuística analisada foi composta de $366(98,7 \%)$ crianças de cor branca e $5(1,3 \%)$ de cor preta. Em relação ao sexo, $207(55,8 \%)$ crianças pertenciam ao masculino, e $164(44,2 \%)$ ao feminino.

A neoplasia maligna primária mais freqüente (Tabela 1) foi a leucemia $(36,6 \%)$, seguida pelos tumores do SNC e miscelânea de neoplasias intracranianas e intra-espinhais $(21 \%)$ e pelos linfomas e neoplasias reticuloendoteliais $(12,9 \%)$.

A neoplasia maligna primária na criança apresentou-se no pré-escolar em $41,5 \%$ (destes, $61,0 \%$ ocorreu no sexo masculino e $39,0 \%$ no feminino); no escolar em $24 \%$ ( $50,6 \%$ no sexo masculino e $49,4 \%$ no feminino); no adolescente em $18,3 \%$ (58,8\% no sexo masculino e $41,2 \%$ no feminino); e no lactente em $16,2 \%$ ( $46,7 \%$ no sexo masculino e $53,3 \%$ no feminino). Não foram observados casos de neoplasia maligna primária no neonato no período estudado.

Tabela 1 - Distribuição, em número e em percentagem, das crianças portadoras de neoplasias malignas primárias, segundo o grupo de diagnóstico e o sexo

\begin{tabular}{lcccc}
\hline \multirow{2}{*}{ Grupo de diagnóstico } & \multicolumn{5}{c}{ Sexo } \\
\cline { 2 - 6 } & \multicolumn{2}{c}{ Masculino } & \multicolumn{2}{c}{ Feminino } \\
& $\mathbf{n}$ & $\mathbf{\%}$ & $\mathbf{n}$ & $\mathbf{\%}$ \\
\hline Leucemia & 72 & 34,7 & 64 & 39,0 \\
Linfomas e neoplasias reticuloendoteliais & 35 & 16,9 & 13 & 7,9 \\
Tumores do SNC* & 43 & 20,8 & 35 & 21,3 \\
Tumores do sistema nervoso simpático & 20 & 9,7 & 6 & 3,7 \\
Retinoblastoma & 4 & 1,9 & 7 & 4,2 \\
Tumores renais & 4 & 1,9 & 9 & 5,5 \\
Tumores hepáticos & 1 & 0,5 & 0 & 0 \\
Tumores ósseos malignos & 8 & 3,9 & 6 & 3,7 \\
Sarcomas de partes moles & 12 & 5,8 & 8 & 4,9 \\
Neoplasias de células germinativas ${ }^{\dagger}$ & 6 & 2,9 & 9 & 5,5 \\
Carcinomas e outras neoplasias malignas epiteliais & 2 & 1,0 & 6 & 3,7 \\
Outros e tumores malignos não especificados & 0 & 0 & 1 & 0,6 \\
\hline Total & 207 & 100,0 & 164 & 100,0 \\
\hline
\end{tabular}

Fonte: SOH/SAME/HIJG, janeiro/1994 a dezembro/1998.

* Tumores do SNC e miscelânea de neoplasias intracranianas e intra-espinhais.

† Neoplasias de células germinativas, trofoblásticas e outras gonadais. 
Os grupos de diagnósticos leucemia, linfomas e neoplasias reticuloendoteliais, tumores do SNC e miscelânea de neoplasias intracranianas e intra-espinhais, tumores do sistema nervoso simpático, retinoblastoma e tumores renais foram mais freqüentes no pré-escolar (Tabela 2).

Neste estudo, foi avaliada a extensão clínica da doença neoplásica maligna primária, em relação ao grupo de diagnóstico. Apresentavam doença localizada, na ocasião do diagnóstico, 104 crianças (44,3\%), e doença não localizada, 131 crianças $(55,7 \%)$. O grupo de diagnóstico linfomas e neoplasias reticuloendoteliais apresentou a maior freqüência de doença não localizada no ingresso ao serviço (Tabela 3).

Correlacionando o status vital com a extensão clínica da doença, observou-se que as crianças com doença localizada encontravam-se vivas, fora de tratamento, em $74,1 \%$ dos casos $(n=77)$; vivas, em tratamento, em 9,6\% $(n=10)$, e $16,3 \%(\mathrm{n}=17)$ foram a óbito. Entre as crianças com doença não localizada, $38,2 \%(\mathrm{n}=50)$ estavam fora de tratamento; $6,1 \%(n=8)$ estavam em tratamento, e $55,7 \%(n=73)$ foram a óbito.

A Figura 1 mostra a representação gráfica da $\mathrm{ACM}$ em um plano com duas dimensões. Observa-se uma clara divisão em dois grupos de variáveis, a qual é norteada pela distância entre os pontos que representam as variáveis categóricas grupo etário, sexo, status vital e extensão clínica da doença. No primeiro grupo, pela proximidade dos pontos, evidencia-se uma associação direta entre doença localizada, vivo, escolar, adolescente e sexo feminino. No segundo grupo, revela-se uma associação direta entre doença não localizada, óbito, lactente, pré-escolar e sexo masculino. A variável lactente encontra-se isolada, caracterizando desta forma, que não houve associação a nenhum dos grupos. Ao incluirmos uma terceira dimensão, esta associação é corroborada e fortalecida, com inércia de 65,88\%.

\section{Discussão}

Os estudos epidemiológicos descritivos, de origem hospitalar, no Brasil, sobre câncer pediátrico, são escas$\operatorname{sos}^{10}$, e inexistem em Santa Catarina. Nas publicações disponíveis no Brasil, foram registradas 259 crianças portadoras de neoplasias malignas, atendidas no Hospital do Câncer A.C. Camargo ${ }^{12}$ (São Paulo), no ano de 1994, e 695 no registro do Hospital Erasto Gaertner ${ }^{16}$ (Curitiba), no período de 1990 a 1999.

Neste estudo, foram analisados 371 dos 376 casos novos de câncer pediátrico do RHC do Hospital Infantil Joana de Gusmão, no período de cinco anos, utilizando-se uma classificação fundamentada e agrupada por tipos histopatológicos baseados na de Marsden 1,14,15,26, que os divide em doze grandes grupos.

Em relação à ocorrência do câncer, descreve-se diferenças geográficas e étnicas nos estudos epidemiológi$\cos ^{3,4,6,7,9}$. Em um registro de câncer realizado em $\mathrm{Cuba}^{11}$, e em outros brasileiros ${ }^{12,16}$, os grupos de diagnósticos mais freqüentes foram leucemia, os linfomas e neoplasias reticuloendoteliais, os tumores do SNC, e miscelânea de neoplasias intracranianas e intra-espinhais. Contrariamente a estes estudos, porém de forma análoga à literatura americana ${ }^{1,27}$, o grupo histológico predominante neste estudo foi a leucemia $(36,6 \%)$, seguido pelos tumores do SNC, pela miscelânea de neoplasias intracranianas e intra-espinhais $(21 \%)$, e pelos linfomas e neoplasias reticuloendoteliais $(12,9 \%)$ (Tabela 1).

A maioria dos estudos aponta para um discreto predomínio no sexo masculino para as neoplasias malignas nas crianças. No entanto, para alguns sítios e/ou tipos histológicos, ao se introduzirem outros fatores, tais como a idade, podem haver diferenças de acordo com o sexo ${ }^{1,27-29}$. Assim, a incidência do câncer em crianças, em Cuba (19861990), revelou predomínio no sexo feminino, observado

Tabela 2 - Distribuição das crianças portadoras de neoplasias malignas primárias, segundo o grupo de diagnóstico e o grupo etário

\begin{tabular}{|c|c|c|c|c|c|c|c|c|c|}
\hline & \multicolumn{8}{|c|}{ Grupo etário } & \multirow{3}{*}{$\begin{array}{c}\text { Total } \\
\mathbf{n}\end{array}$} \\
\hline & \multicolumn{2}{|c|}{ Lactente } & \multicolumn{2}{|c|}{ Pré-escolar } & \multicolumn{2}{|c|}{ Escolar } & \multicolumn{2}{|c|}{ Adolescente } & \\
\hline & n & $\%$ & $\mathbf{n}$ & $\%$ & $\mathbf{n}$ & $\%$ & $\mathbf{n}$ & $\%$ & \\
\hline Leucemia & 19 & 14,0 & 67 & 49,3 & 31 & 22,7 & 19 & 14,0 & 136 \\
\hline Linfomas e neoplasias reticuloendoteliais & 5 & 10,4 & 19 & 39,6 & 13 & 27,1 & 11 & 22,9 & 48 \\
\hline Tumores do SNC* & 8 & 10,2 & 28 & 36,0 & 25 & 32,0 & 17 & 21,8 & 78 \\
\hline Tumores do sistema nervoso simpático & 9 & 34,6 & 14 & 53,9 & 1 & 3,8 & 2 & 7,7 & 26 \\
\hline Retinoblastoma & 4 & 36,4 & 5 & 45,4 & 2 & 18,2 & 0 & 0 & 11 \\
\hline Tumores renais & 3 & 23,1 & 9 & 69,2 & 0 & 0 & 1 & 7,7 & 13 \\
\hline Tumores hepáticos & 1 & 100,0 & 0 & 0 & 0 & 0 & 0 & 0 & 1 \\
\hline Tumores ósseos malignos & 2 & 14,3 & 2 & 14,3 & 5 & 35,7 & 5 & 35,7 & 14 \\
\hline Sarcomas de partes moles & 2 & 10,0 & 5 & 25,0 & 7 & 35,0 & 6 & 30,0 & 20 \\
\hline Neoplasias de células germinativas ${ }^{\dagger}$ & 5 & 33,3 & 3 & 20,0 & 4 & 26,7 & 3 & 20,0 & 15 \\
\hline Carcinomas e outras neoplasias malignas epiteliais & 2 & 25,0 & 2 & 25,0 & 1 & 12,5 & 3 & 37,5 & 8 \\
\hline Outros e tumores malignos não especificados & 0 & 0 & 0 & 0 & 0 & 0 & 1 & 100,0 & 1 \\
\hline
\end{tabular}

Fonte: SOH/SAME/HIJG, janeiro/1994 a dezembro/1998.

† Neoplasias de células germinativas, trofoblásticas e outras gonadais. 


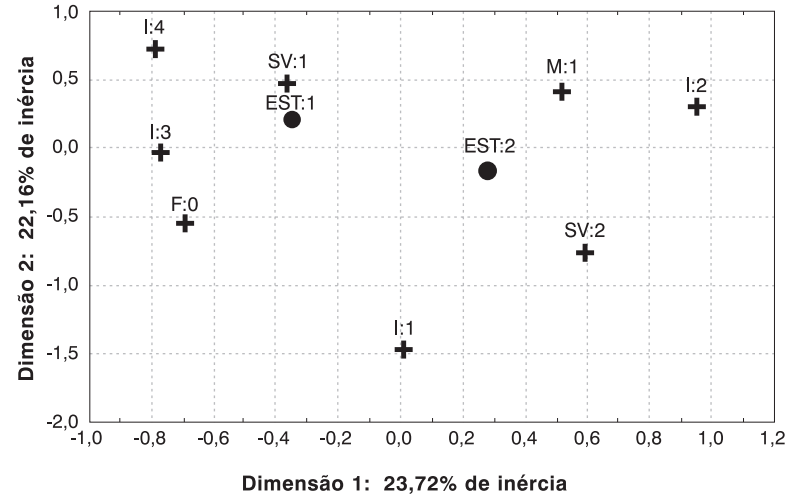

\begin{tabular}{|c|c|c|c|c|}
\hline \multirow[t]{5}{*}{ Legenda: } & $1: 1$ & Lactente & $\mathrm{M}: 1$ & Sexo masculino \\
\hline & $1: 2$ & Pré-escolar & SV:1 & Vivo \\
\hline & $1: 3$ & Escolar & SV:2 & Óbito \\
\hline & $1: 4$ & Adolescente & EST:1 & Doença localizada \\
\hline & $\mathrm{F}: 0$ & Sexo feminino & EST:2 & Doença não localizada \\
\hline
\end{tabular}

Figura 1 - Análise de correspondência múltipla para as variáveis categóricas grupo etário, sexo, status vital e extensão clínica das neoplasias malignas primárias. SOH/SAME/HIJG, janeiro/1994 a julho/2000

principalmente para os tumores do sistema nervoso simpático, retinoblastoma e tumores renais ${ }^{11}$.

No Brasil, na cidade de Goiânia, em um estudo de base populacional de câncer pediátrico ${ }^{30}$, referente ao período de 1989 a 1996, foi observado predomínio no sexo feminino. A autora atribuiu a decorrência deste aumento aos riscos aumentados encontrados para os tumores renais, os tumores malignos dos ossos e sarcomas de partes moles, naquela região ${ }^{30}$.

A publicação realizada pela Liga Paranaense de Combate ao Câncer (1990-99) ${ }^{16}$ registrou, no sexo masculino, percentual de $60,9 \%$, e descreveu predomínio no sexo feminino para os tumores de células germinativas ${ }^{16}$.
No presente estudo, as neoplasias malignas também ocorreram com maior freqüência no sexo masculino $(55,8 \%)$. No entanto, devido ao tamanho da amostra, não foi possível estabelecer novas relações quanto ao sexo e ao grupo de diagnóstico pela introdução de outras variáveis. Em relação à raça, foram registrados somente 5 casos $(1,3 \%)$ de neoplasia maligna primária em crianças de cor preta, não podendo, conseqüentemente, obter-se comparações entre as raças.

Analisando a faixa etária de ocorrência do câncer, um estudo realizado no período de 1990 a 1995 pelo Programa de Vigilância, Epidemiologia e Resultados Finais (SEER), do Instituto Nacional de Câncer dos Estados Unidos da América (EUA), observou que a mais alta taxa de incidência ocorreu nas crianças menores de cinco anos de idade ${ }^{1}$. No registro de câncer de $\mathrm{Cuba}^{11}$, bem como no da cidade de Goiânia $^{30}$ e em outros registros brasileiros ${ }^{12,16}$, ocorreu também predomínio em menores de cinco anos de idade.

Neste registro, o grupo etário pré-escolar foi também o mais suscetível ao desenvolvimento do câncer. Observouse, entretanto, variação da percentagem relativa de acordo com o grupo etário, quando comparado com o grupo de diagnóstico. A leucemia foi o diagnóstico mais comum em todos os grupos etários, todavia a percentagem relativa foi maior no pré-escolar (49,3\%). Concordando com outros registros $1,11,12,16$, os linfomas e neoplasias reticuloendoteliais, os tumores do SNC, e miscelânea de neoplasias intracranianas e intra-espinhais ocorreram com maior freqüência no pré-escolar e no escolar. Os tumores do sistema nervoso simpático, retinoblastoma e tumores renais ocorreram predominantemente no lactente e no pré-escolar. Os tumores ósseos malignos foram mais freqüentes nos grupos etários escolar e adolescente, e os sarcomas de partes moles, no pré-escolar, escolar e adolescente. A baixa freqüência observada nos demais grupos de diagnóstico impossibilitou uma adequada avaliação em relação ao grupo etário (Tabela 2).

Tabela 3 - Distribuição das crianças portadoras de neoplasias malignas primárias, segundo o grupo de diagnóstico e a extensão clínica da doença

\begin{tabular}{|c|c|c|c|c|c|}
\hline \multirow{3}{*}{ Grupo de diagnóstico } & \multicolumn{4}{|c|}{ Extensão clínica da doença } & \multirow{3}{*}{$\begin{array}{c}\text { Total } \\
\text { n }\end{array}$} \\
\hline & \multicolumn{2}{|c|}{ Localizada } & \multicolumn{2}{|c|}{ Não localizada } & \\
\hline & $\mathbf{n}$ & $\%$ & $\mathbf{n}$ & $\%$ & \\
\hline Linfomas e neoplasias reticuloendoteliais & 7 & 14,6 & 41 & 85,4 & 48 \\
\hline Tumores do SNC* & 50 & 64,1 & 28 & 35,9 & 78 \\
\hline Tumores do sistema nervoso simpático & 6 & 23,1 & 20 & 76,9 & 26 \\
\hline Retinoblastoma & 7 & 63,6 & 4 & 36,4 & 11 \\
\hline Tumores renais & 5 & 38,5 & 8 & 61,5 & 13 \\
\hline Tumores hepáticos & 0 & 0 & 1 & 100,0 & 1 \\
\hline Tumores ósseos malignos & 9 & 64,3 & 5 & 35,7 & 14 \\
\hline Sarcomas de partes moles & 8 & 40,0 & 12 & 60,0 & 20 \\
\hline Neoplasias de células germinativas ${ }^{\dagger}$ & 8 & 53,3 & 7 & 46,7 & 15 \\
\hline Carcinomas e outras neoplasias malignas epiteliais & 3 & 37,5 & 5 & 62,5 & 8 \\
\hline Outros e tumores malignos não especificados & 1 & 100,0 & 0 & 0 & 1 \\
\hline
\end{tabular}

Fonte: SOH/SAME/HIJG, janeiro/1994 a dezembro/1998. * * Tumores do SNC e miscelânea de neoplasias intracranianas e intra-espinhais. $\dagger$ Neoplasias de células germinativas, trofoblásticas e outras gonadais. 
Em relação ao câncer, é necessário ao profissional da saúde estar atento ao diagnóstico precoce, para detectar a doença ainda em uma fase localizada, assim como para o rápido encaminhamento aos centros oncológicos pediátricos especializados ${ }^{7,26}$.

No registro de câncer realizado no Paraná ${ }^{16}$, foi descrita percentagem de $49,4 \%$ de doença não localizada, ao ingresso hospitalar, caracterizando diagnóstico tardio. Os grupos de diagnóstico com percentuais mais elevados de doença não localizada foram os tumores do sistema nervoso simpático, sarcoma de partes moles, tumores renais, linfomas e neoplasias reticuloendoteliais ${ }^{16}$.

$\mathrm{Na}$ ocasião do diagnóstico, neste estudo, 104 crianças $(44,3 \%)$ apresentavam doença localizada, e $131(55,7 \%)$, doença não localizada. O grupo de diagnóstico linfomas e neoplasias reticuloendoteliais e o grupo dos tumores do sistema nervoso simpático apresentaram as maiores freqüências de doença não localizada, na ocasião do diagnóstico (Tabela 3).

Avaliando o status vital das crianças portadoras de neoplasias malignas primárias em relação à extensão clínica da doença, verificou-se que, das crianças com doença localizada, $83,7 \%$ estavam vivas e $16,3 \%$ foram a óbito. Nas crianças com doença não localizada, somente $44,3 \%$ estavam vivas, e 55,7\% foram a óbito (Tabela 3 ), enfatizando a importância do diagnóstico precoce.

Na busca de um padrão de associação entre as variáveis categóricas, utilizando-se a ACM (Figura 1), entre outras ocorrências verificadas ${ }^{31}$, poderíamos concluir que há uma associação direta entre doença localizada, vivo, escolar, adolescente e sexo feminino. Ressalta-se que a evidência de um padrão de associação não se constitui em uma relação causal. Estes resultados revelam a necessidade de se realizar estudos analíticos multicêntricos, visando à confirmação da ocorrência destas associações, e assim estabelecer fatores de risco para o câncer pediátrico.

\section{Referências bibliográficas}

1. Ries LAG, Smith MA, Gurney JG, Linet M, Tamra T, Young JL, et al. Cancer incidence and survival among children and adolescents: United States SEER Program 1975-1995. Bethesda (MD): National Cancer Institute; 1999.

2. Lukens JN. Progress resulting from clinical trials. Solid tumors in childhood cancer. Cancer Supplement 1994;74:2710-18.

3. Ferreira RM, Fernandes PL, Pinheiro LR. Registro de câncer de base populacional: uma proposta para a apresentação dos dados pediátricos brasileiros. Rev Bras Cancerol 1997;43:133-37.

4. Quintana J. Tumores cancerosos más frecuentes em pediatria. Del oncólogo al pediatra. Pediatria Al Dia 1998;14:251-58.

5. American Academy of Pediatrics Section Statement. Guidelines for the pediatric cancer center and role of such centers in diagnosis and treatment. Pediatrics 1997;99:139-41.

6. Franco EL. Cancer epidemiology: substance and methods. Revista Ciência e Cultura 1994;46:46-62.

7. Barr RD. The challenge of childhood cancer in the developing world. East Afr Med J 1994;71:223-5.

8. Hamada GS, Cerny CA, Ribeiro KCB, Tagawa EK, Alcântara OS, Neuenschwander R, et al. Implantação do registro hospitalar de câncer e a qualidade das informações - Hospital A.C. Camargo. Acta Oncol Bras 1995;15:202-6.
9. Mirra AP. Registros de câncer na América Latina. Rev Bras Cancerol 1997;43:1-11.

10. Eichenberg A, Kapees DS, Petry C, Silva A, Tatsch P, Jacques L, et al. Características dos registros de câncer e o impacto potencial no controle da doença no Brasil. R Med PUCRS 1999;9:11-17.

11. Parkin DM, Luir CS, Whelan SL, Gao Y-T, Ferlay J, Powel J. Cancer incidence on five continents-Cuba. IARC Sci Publ 1992;120:210-13.

12. Centro de Tratamento e Pesquisa Hospital do Câncer A.C. Camargo. Registro hospitalar de câncer 1994. Disponível em http:// www.hcanc.org.br

13. Ministério da Saúde. Conselho Nacional de Saúde. Resolução n. 251 de 05/08/97. Diretrizes e normas regulamentadoras de pesquisa envolvendo seres humanos [site na Internet]. Disponível em http:// www.cepsh.ufsc.br/originais/Res25197.doc. Acessado 26 de setembro de 2002.

14. Marsden HB. The classification of childhood tumours. IARC Sci Publ 1988;(87):9-16.

15. Birch JM, Marsden HB. A classification scheme for childhood cancer. Int J Cancer 1987;40:620-4.

16. Liga Paranaense de Combate ao Câncer. Câncer na infância e na adolescência. Curitiba: LPCC; 2000.p.20.

17. Rosai J. Ackerman's surgical pathology. $7^{\text {a }}$ ed. St. Louis: CV Mosby; 1989.

18. Lukens JN. Classificação e diferenciação das leucemias agudas. In: Lee GR, Bithell TC, Foerster J, Athens JW, Lukens JN. Wintrobe hematologia clínica. $1^{\mathrm{a}}$ ed. São Paulo: Manole; 1998. v.2 p.2062-82.

19. Percy C, Holten VV, Muir C. Classificação Internacional de Doenças para Oncologia. In: Organização Mundial da Saúde. $2^{\mathrm{a}}$ ed. São Paulo: Editora da Universidade de São Paulo; 1996.

20. Kramárová E, Stiller CA, Ferlay J, Parkin DM, Draper GJ, Michaelis $\mathrm{J}$, et al. International Classification of Childhood Cancer. Int $\mathrm{J}$ Cancer 1996;68:759-65.

21. Hudson MM, Donaldson SS. Hodgkin's disease. In: Pizzo PA, Poplack DG, editores. Principles and practice of pediatrics oncology. $3^{\mathrm{a}}$ ed. Philadelphia: Lippincott-Haven; 1997. p.523-43.

22. Instituto Brasileiro de Geografia e Estatística - IBGE. Departamento de População e Indicadores Sociais. Síntese de indicadores sociais 1999. Estudos e Pesquisas. Informação demográfica e socioeconômica 2000;4:197.

23. Marcondes E, Machado DVM, Setian N, Carrazza FR. Crescimento e desenvolvimento. In: Marcondes E. Pediatria básica. $8^{\mathrm{a}}$ ed. São Paulo: Sarvier; 1994.p.35-63.

24. Pereira MG. Métodos empregados em epidemiologia. In: Epidemiologia: teoria e prática. Rio de Janeiro: Guanabara Koogan; 1995.p.269-88.

25. Carrol JD, Green PE, Schaffer CM. Interpoint distance comparisons in correspondence analysis. J Marketing Res 1986;23:271-80.

26. Petrilli AS, Carneiro JL, Cypriano M, Angel A, Toledo S. Diferenças clínicas, epidemiológicas e biológicas entre o câncer na criança e no adulto. Rev Bras Cancerol 1997;43:191-203.

27. International Incidence of Childhood Cancer, Vol. II. IARC Sci Publ 1998;(144):1-391.

28. Bunin GR, Feuer EJ, Witman PA, Meadows AT. Increasing incidence of childhood cancer: report of 20 years experience from the Greater Delaware Valley Pediatric Tumor Registry. Paediatr Perinat Epidemiol 1996;10:319-38

29. Bleyer WA. What can be learned about childhood cancer from Cancer Statistics Review 1973-1988. Cancer 1993;71:3229-36.

30. Braga PE. Câncer na infância: tendências e análise de sobrevida em Goiânia (1989-1996) [dissertação]. São Paulo (SP): Faculdade de Saúde Pública da Universidade de São Paulo; 2000.

31. Silva DB. Câncer pediátrico: análise do registro hospitalar de um centro de referência de Santa Catarina [dissertação]. Florianópolis (SC): Centro de Ciências Médicas da Universidade Federal de Santa Catarina; 2001.

Endereço para correspondência:

Dra. Denise Bousfield da Silva

Rua Rui Barbosa, 154 - Agronômica

CEP 88025-301 - Florianópolis, SC 